lait et la récupération de ${ }^{15} \mathrm{~N}$ dans ces produits sont suivis durant 6 j, 2 fois par jour, au moment des traites ( $8 \mathrm{~h}$ et $17 \mathrm{~h}$ ). L'enrichissement de l'azote non protéique du lait et du sang est suivi de la même manière.

Le bilan azoté, mesuré sur 6 j, montre que sur les $30,0 \mathrm{~g}$ d'azote ingérés par jour $(100 \%)$, $12,3 \mathrm{~g}$ sont retrouvés dans les fèces $(41 \%), 9,9 \mathrm{~g}$ dans l'urine $(33 \%)$ et $9,8 \mathrm{~g}$ dans le lait $(33 \%)$. Le bilan de l'azote est légèrement négatif $(-2,0 \mathrm{~g} / \mathrm{j})$.

La proportion ${ }^{15} \mathrm{~N} / \mathrm{N}$ des produits étudiés passe par un maximum à $24 \mathrm{~h}$ (le matin de $\mathrm{J} 1$ ) dans les fractions ANP du lait $(0,50 \%)$ et du sang $(0,58 \%)$. Pour les fèces $(0,64 \%)$, l'urine $(0,59 \%)$ et le lait $(0,51 \%)$, le maximum s'observe dans le troisième prélèvement (le soir de $\mathrm{J} 1$ ). L'urine se distingue cependant par une augmentation très précoce de son enrichissement en ${ }^{15} \mathrm{~N}$. Après ce maximum, la baisse de concentration est régulière et de plus en plus lente. Les proportions $15 \mathrm{~N} / \mathrm{N}$ résiduelles en fin d'expérience, 5 à $6 \mathrm{j}$ après la distribution du foin marqué, sont peu différentes entre tous ces produits (environ $0,4 \%$ ) et très proches de la concentration naturelle $(0,37 \%)$.

L'ingestion de $15 \mathrm{~N}$ au dessus de la teneur naturelle est de $281 \mathrm{mg} \mathrm{(100 \% )} \mathrm{dont} 74 \mathrm{mg}$ sont récupérés dans les fèces (26\%), $44 \mathrm{mg}$ dans l'urine $(15 \%)$ et $32 \mathrm{mg}$ dans le lait $(11 \%)$. L'essentiel de la récupération de $15 \mathrm{~N}(75 \%)$ a lieu dans les 3 premiers jours. $131 \mathrm{mg}$ de ${ }^{15} \mathrm{~N}(47 \%$ de l'ingéré) ne sont pas récupérés dans la durée de l'expérience et peuvent être considérés comme intégrés dans le pool azoté de l'organisme. Cette observation confirme la forte proportion de l'azote digéré qui participe au renouvellement du pool azoté de l'animal (estimée à environ $70 \%$ ).

\section{Paramètres sanguins et bilan azoté chez le taurillon culard en engraissement intensif ou en croissance faible suivie de croissance accélérée*. C Van} Eenaeme, JL Hornick, A Clinquart, N Korsack, L Istasse (Service de nutrition B43, faculté de médecine vétérinaire, université de Liège, 4000 Liège-Sart Tilman, Belgique)

L'état métabolique a été mesuré, d'une part, sur 4 taurillons culards Blanc-Bleu-Belge, soumis pendant 4 mois à une croissance faible de $0,57 \pm$ $0,01 \mathrm{~kg} / \mathrm{j}$ (CF) suivie d'une période de croissance accélérée de 1,57 $\pm 0,09 \mathrm{~kg} / \mathrm{j}$ (CA) et, d'autre part, sur un groupe témoin de 4 animaux en engraissement intensif continu $(1,28 \pm 0,11 \mathrm{~kg} / \mathrm{j})$. Pendant la $1^{\text {re }}$ période l'urémie pré-prandiale était significativement $(P<0,001)$ plus basse dans le groupe CF que dans le groupe témoin ; $72,7 \pm$ 12,9 contre $138,4 \pm 12,1 \mathrm{mgN} / \mathrm{l}$. Le passage à l'engraissement a fait augmenter l'urémie qui a atteint celle des témoins en fin de période (227 mg $/ /$ ). En revanche, le taux de créatinine a été plus élevé dans le groupe $\mathrm{CF}$ en première période : $23,9 \pm 2,6$ contre $19,5 \pm 1,9 \mathrm{mg} / \mathrm{l} \mathrm{chez}$ les témoins. Pendant la période de croissance compensatrice la créatininémie a diminué dans le groupe CF et a rejoint en fin de période celle du groupe témoin, pour lequel on a observé une augmentation continue suivie d'un plateau en fin d'engraissement. La glycémie était significativement plus faible $(P<0,01)$ en CF : $824,3 \pm 63,0$ contre $953,2 \pm 55,5 \mathrm{mg} / \mathrm{l}$ chez les témoins. Ceci pourrait être imputé à une diminution de la néoglucogenèse hépatique résultant d'un apport diminué de propionate par suite d'un niveau de fermentation plus faible dans le rumen. Elle a augmenté légèrement en période CA et s'est située à un niveau plus élevé que celle des témoins pour lesquels on a observé une diminution régulière dans le temps : $820,0 \pm 46,7$ vs $770,7 \pm 48,3(P<0,05)$ respectivement pour le groupe CA et témoin. L'azote $\alpha$-aminé était plus bas en CF : $40,29 \pm 2,71$ vs $44,57 \pm 2,42 \mathrm{mgN} / \mathrm{l}$ $(P<0,01)$ pour les témoins, et a augmenté vers la fin pour atteindre $53 \mathrm{mgN} / \mathrm{l}$ dans les 2 groupes.

Le bilan azoté a été mesuré au milieu de la période $C F, 2 \mathrm{sem}$ après le passage à la ration riche et 1 mois avant l'abattage. En période CF la rétention azotée a été significativement plus basse $(P<0,001)$ pour le groupe $\mathrm{CF}: 21,3 \pm 4,9 \mathrm{~g} \mathrm{~N} / \mathrm{j}$ que celle du groupe témoin $61,4 \pm 2,5 \mathrm{~g} \mathrm{~N} / \mathrm{j}$. Elle a rejoint celle des témoins et est restée à ce niveau pendant les périodes ultérieures, à savoir : $67,5 \pm 17,8$ pour CF et $62,4 \pm 9,9$ pour les témoins en période 2 , et $57,9 \pm 1,5$ pour $\mathrm{CF}$ contre $61,5 \pm 10,7 \mathrm{~g} \mathrm{~N} / \mathrm{j}$ pour les témoins un mois avant l'abattage. L'azote retenu par $\mathrm{kg}$ de gain de poids vif se situait aux environs de $40 \mathrm{~g}$ par jour et était identique dans les 2 groupes, ce qui indique que le rendement de dépôt azoté restait inchangé.

II semble donc qu'une période de croissance faible $(0,5 \mathrm{~kg} / \mathrm{j})$ de 4 mois n'a pas eu d'effet négatif irréversible sur les performances des animaux comme en témoignent l'efficience de la fixation d'azote (bilan N, urémie) ainsi que l'état d'approvisionnement de l'énergie (glycémie).

\footnotetext{
* Recherches subventionnées par I'IRSIA, Bruxelles, Belgique
} 